\title{
On-Line 3D Geometric Model Reconstruction
}

\author{
H. Zolfaghari ${ }^{1}$ and K. Khalili ${ }^{2}$ \\ ${ }^{1}$ Computer Engineering Department, Islamic Azad University, Birjand Branch, Birjand, Iran \\ h.zolfagari@gmail.com \\ ${ }^{2}$ Mechanical Engineering Department, University of Birjand, Iran \\ kkhalili@birjand.ac.ir
}

\begin{abstract}
Triangulation techniques along with laser technology have been widely used for 3D scanning, however, 3D scanning of moving object and online modeling have received less attention. The current work describes a system developed for on-line CAD model generation using scanned data. The system developed uses structured laser light pattern and a digital CCD camera to generate and capture images from which the range data is extracted. The data is then analyzed to reconstruct the geometric model of the moving object. To exploit the potential of a geometric modeler the model is represented in a commercial CAD software. To reduce errors the system employs on-line calibration, light distribution correction algorithm, camera calibration, and subpixeling techniques. A data reduction scheme has also been incorporated to eliminate redundant data.
\end{abstract}

Keywords: 3D Modeling, 3D Scanning, Surface Reconstruction. On-line Model Generation.

\section{Introduction}

3D scanning is an interesting subject and of interest to many researchers. During the past few years much effort has been devoted to technology development and improving the accuracy and speed of 3D scanning while reducing the cost of such systems. The potential areas of applications include reverse engineering, product development, quality control, and 3D photography. The general procedure in 3D scanning consists of scanning an object, usually with a range sensor, merging several data sets into a single registered data set, and representing the data set in a computer representation form, such as a mesh or a set of surfaces.

Generally, the methods of measurement of three-dimensional geometry are divided into two main methods, i.e. tactile measurement and optical measurement [1].

Contact methods based on mechanical sensors have been used widely for accurate industrial applications; however the accuracy, speed, cost and being contact of such systems are of concern [2].

Non-contact methods use different technologies amongst which optical methods including active and passive systems are the most widely used methods. Triangulation technique along with laser technology provides a relatively accurate, fast, and inexpensive system for 3D scanning. Passive stereo vision is attractive in being a passive method but current systems lack the accuracy required for the most of industrial applications. 
Currently, many 3D scanners use structured light technique using laser light source. Structured light is the projection of a light pattern such as a plane, grid, or more complex shape at a known angle onto an object. Use of a beam of light and fannig it out into a sheet-of-light is widely used in 3D scanning. When the sheet-of-light intersects with the object of interest, a bright line of light can be seen on the surface of the object. By viewing this line of light, the observed deviation of the line from base line can be translated into height variations [3]; Fig.1.

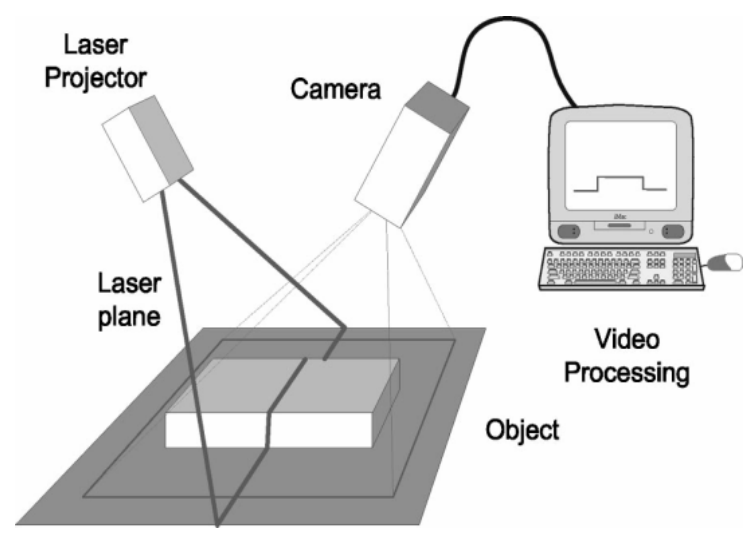

Fig. 1. Active optical triangulation technique

Although 3D scanning has received a great attention from research community but little works have been carried out to on-line CAD model generation of moving objects. The potential applications include 3D modeling of continuous production line such as extruded products [4].

This paper deals with on-line CAD model generation of scanned data. The system developed uses structured laser light pattern and a digital CCD camera to generate and capture images from which the range data is extracted. The data is then analyzed to reconstruct the geometric model of the moving object. Finally, the model is represented in a commercial CAD software.

\section{Conceptual Design}

In scanning process a set of 3D points from the measured object surface is acquired then transformed by software into a geometric model of the object. The accuracy of the model depends primarily on the resolution of scanning; i.e. the distance between adjacent scanned points. Moreover, the mapping process in which the 3D coordinate of the object is transformed into 2D coordinate of image has to be precise and accurate. Factors such as the resolution of the imaging device, the thickness and more importantly the sharpness of the light line and its uniformity, adjustment and calibration of the light source and the imaging device, the environmental factors and object color and texture, and the data extraction algorithm 
affect the accuracy of acquired data. The system has to be designed such that the final accuracy required is obtained.

The current work describes an inexpensive 3D scanner using off-the-shelf components and software. In system design emphasis is given to modularity, use of off-the-shelf components, full potential use of an existing commercial CAD software, flexibility, and reduced cost.

The designed system contains four modules; lighting module, imaging module, processing module, and display module. The system must be able to generate on-line 3D models of moving objects as they pass under the scanner.

\section{Previous Works}

Three dimensional scanning has received great attention in research community. These include contact methods and non-contact methods. Coordinate Measuring Machine $(\mathrm{CMM})$ is the most accurate mechanical machine being in use for decades. There are currently many commercial systems in the market. They are mainly used for dimensional quality control but they are also used for 3D scanning, but the process is very time consuming. Non-contact methods especially optical methods including passive and active techniques are used widely and research works towards increasing accuracy and speed, reduced cost, ease of use, are in progress.

Son et al [1] developed a 3D scanner using structured light. They tried to automate the whole process and eliminate the need for manual operations. Lombardo et al [3] developed a time of scan triangulation technique. An accuracy of $100 \mu \mathrm{m}$, a working distance of $20 \mathrm{~cm}$ and a very limited range of $10 \mathrm{~mm}$ has been reported. The accuracy of the system developed depends on the instability of the rotation speed of the mechanical scanner that affects the measurement of the scanning time. Zhang and Wee [5] developed a novel calibration approach to improve the accuracy of the system. They applied back propagation (BP) neural network to the calibration of structured light 3D vision inspection. An accuracy of $0.083 \mathrm{~mm}$ has been reported.

Zexiao et al [6] developed a multi-probe 3D measurement system using CMM machine with capability of structure light scanning to increase the capabilities and flexibility of CMM. A comprehensive review of range sensor development is carried out by Blais F. [7] to which readers are referred to.

Also there are several methods for modeling an object surface. For a review on methods of modeling see [8].

\section{System Development}

The lighting module includes a laser diode UHL 5-10G-G35-90 with visible $635 \mathrm{~nm}$ wavelength. A cylindrical lens is used to fan out the spot beam into a single sheet of light pattern. The adjustment of light source is crucial to the accuracy of final result. To be able to adjust the light source the laser head was mounted on a robot wrist. The robot had 5 degrees of freedom (dof) including wrist 2 dof. This allowed centering the light line and fine adjustment of laser head orientation. To obtain the orientation of laser head, the method developed by [10] was used. 
The light had a Gaussian distribution resulting in an uneven thickness when imaging (1).

$$
\begin{aligned}
& f(x ; \mu, \beta)=\sqrt{\frac{\beta}{2 \pi}} x^{\frac{-3}{2}} \exp \left[\frac{-\beta(x-\mu)^{2}}{2 \mu^{2} x}\right] \quad x>0 \\
& =0 \quad \text { elsewhere }
\end{aligned}
$$

The line of light was corrected by applying inverse distribution function. This provided a relatively uniform line pattern.

The imaging module uses a Canon A640 digital camera. The camera is controlled via computer to take pictures at set intervals of time. The camera is mounted above a conveyor belt. The object is positioned on conveyor belt and moved along with a known and adjustable speed; Fig. 2. The resolution of picture along the movement direction is directly determined by time intervals of picture grabbing and the speed of conveyor and these have to be known precisely.

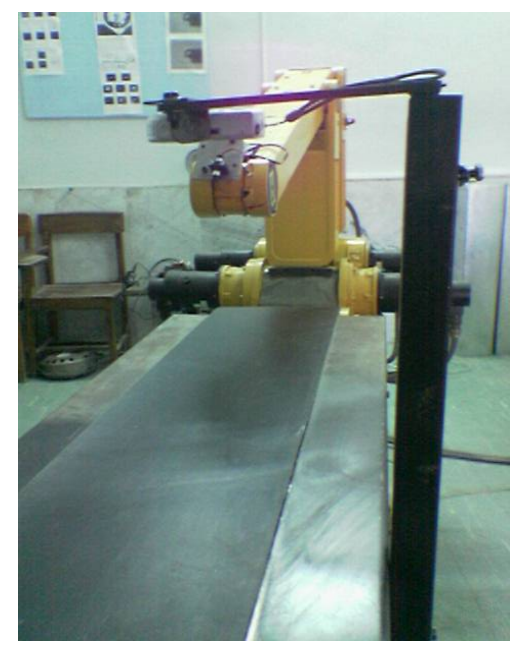

Fig. 2. The laboratory system developed

Prior to use, the camera was calibrated using Tsai method [9].

Processing Module uses MATLAB environment to preprocess and process data. The steps are as follows

- Image capture (Fig. 3)

- Image preprocessing and processing including;

- Grey level conversion

- Object detection

- Edge detection (Fig. 4)

- Image enhancement

- Image segmentation

- $\quad$ Line thinning 
- Base-line detection and construction (Fig. 5)

- Range calculation

- On-line Calibration

- Data points formatting (DXF format)

- Surface reconstruction (Fig. 6)

- Data export

- Model Display

As the color information is not required and to reduce the data, the color image is converted to a grey level image. It is further converted to binary image but the grey level values are kept for downstream use. Noise that is of Salt type is removed using Erosion technique. A structuring element (SE) of size 10 by 10 was used. SE size is important to eliminate noise not the data points.

The width of the line is the distance between the rising edge and falling edge of the line along its width and the position of the line. To locate the position of the line more accurately subpixeling technique was used. To be able to use the accuracy of subpixeling the grey level of edges found and inner pixels were retrieved. Using subpixeling, the centre of the line was calculated and considered as data points. The line was thinned out to a line with a width of a single pixel.

The base line was detected with the same algorithm. A straight line was curvefitted to the base line points. By calculating the distance between data points and the base line and knowing the camera-light source angle the depth information was then extracted using triangulation technique. Using on-line calibration developed by [4] the depth information was transformed into physical depth data.

\section{3D Surface Reconstruction}

At the end of the scanning procedure, a set of 3D coordinates of the different points, $\mathrm{d}_{\mathrm{i}}=\left\{\mathrm{x}_{\mathrm{i}}, \mathrm{y}_{\mathrm{i}}, \mathrm{z}_{\mathrm{i}}\right\} \in \mathrm{R}^{3}$ on the surface $\mathrm{S}_{\mathrm{o}}$ of the object is obtained. The data set $D=\left\{d_{1}, \ldots, d_{n}\right\}$ consists of completely unstructured data. The data points $d_{i}$ 's are arranged as a 3D matrix in which the $x$ and $y$ values are extracted from the grey image and the depth is extracted using the algorithm explained above. The data

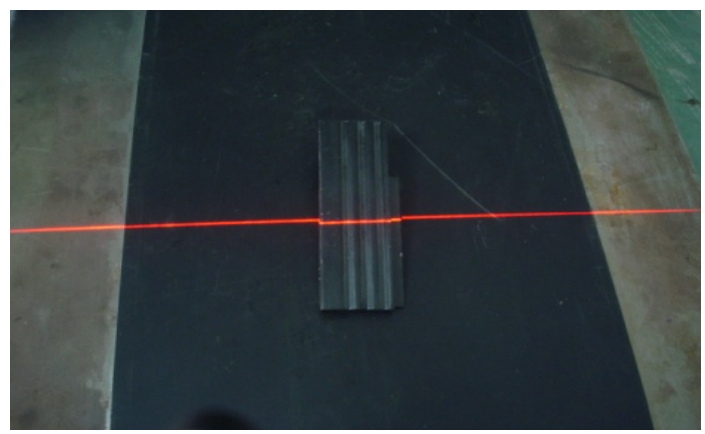

Fig. 3. Object scanning 


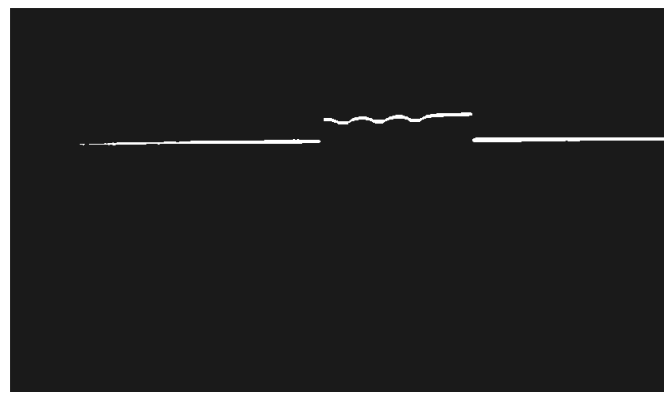

Fig. 4. Edge detection

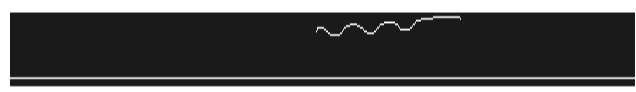

Fig. 5. Image after preprocessing

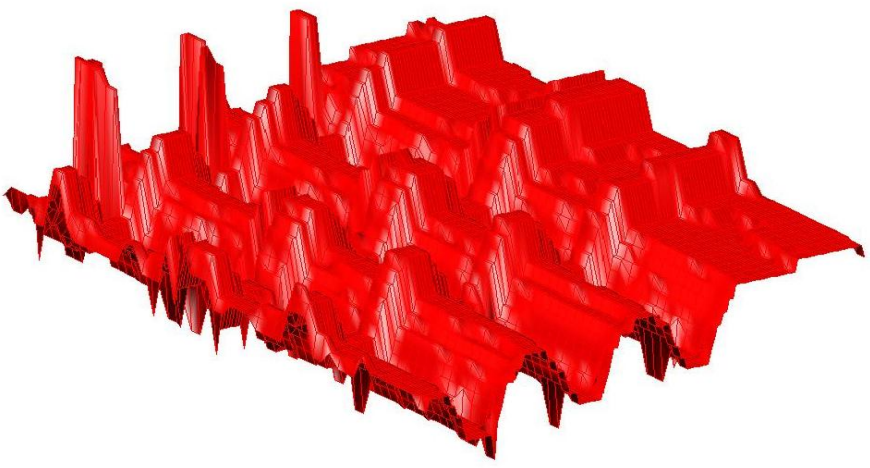

Fig. 6. Surface Reconstruction in AutoCAD

points are arranged in a left to right, top-down order. To decrease the amount of data a data reduction algorithm was employed. The data reduction algorithm eliminates, within a row, the intermediate points with the same depth. The final data points are stored and exported to AutoCAD in DXF format.

3D Mesh was used to reconstruct the initial rough surface. Piecewise 3D mesh of points are generated and using $\mathrm{C} 1$ continuity the pieces are patched together to generate the whole surface.

\section{Discussion and Conclusion}

A simple 3D scanning was developed using off-the-shelf components. The system is capable of generating models of $0.5 * 0.1 * 0.25$ millimeter $\left(\mathrm{L}^{*} \mathrm{~W}^{*} \mathrm{H}\right)$ in resolution. The model is displayed in AutoCAD automatically. It can also be selected to view the 
model within MATLAB. The system benefits from full potential of AutoCAD geometric modeling software. The scanning and surface construction is performed automatically. Use of on-line calibration reduced the systematic errors significantly. Although a data reduction scheme was successfully employed but further works required to improve the process of patching pieces together and reducing more data. The system is used as a stationary single-view system, hence limitation on generating full actual models. To be able to generate complete 3D models several scans have to be performed either by relative motion of scanner-object or preferably using two scanners at sides. The system was tested for a simulated tire tread production line where the thickness of the tread has to be measured and displayed.

\section{References}

1. Son, S., Park, H., Lee, K.H.: Automated Laser Scanning System for Reverse Engineering and Inspection. International Journal of Machine Tools \& Manufacture 42, 889-897 (2002)

2. Zhao, D., Li, S.: A 3D Image Processing Method for Manufacturing Process Automation. Computers in Industry 56, 975-985 (2005)

3. Lombardoa, V., Marzullib, T., Pappalettereb, C., Sforzaa, P.: A Time-Of-Scan Laser Triangulation Technique for Distance Measurements. Optics and Lasers in Engineering 39, 247-254 (2003)

4. Khalili, K., Nazemsadat, S.M.: Improved Accuracy Of On-Line Tire Profile Measurement Using A Novel On-Line Calibration. In: Proc. of the 7th IASTED International Conference on Visualization, Imaging, and Image Processing, Spain, pp. 123-128 (2007)

5. Zhang, Y.: Research In to the Engineering Application of Reverse Engineering Technology. Journal of Materials Processing Technology 139(1-3), 472-475 (2003)

6. Zexiao, X., Jianguo, W., Qiumei, Z.: Complete 3D Measurement in Reverse Engineering Using a Multi-Probe System. International Journal of Machine Tools and Manufacture 45(12-13), 1474-1486 (2005)

7. Blais, F.: Review of 20 Years of Range Sensor Development. Journal of Electronic Imaging 13(1), 231-240 (2004)

8. Campbell, R.J., Flynn, P.J.: A Survey of Free-Form Object Representation and Recognition Techniques. Computer Vision and Image Understanding 81, 166-210 (2001)

9. Tsai, R.Y.: A Versatile Camera Calibration Technique for High- Accuracy 3D Machine Vision Metrology Using Off-the-Shelf TV Cameras and Lenses. IEEE Journal of Robotics and Automation RA-3(4), 323-344 (1987)

10. Nazemsadat, S.M.: On-Line 3D Scanning. MSc. Dissertation, University of Birjand, Iran (2006)

11. Izquierdo, M.A.G., Sanchez, M.T., Ibanez, A., Ullate, L.G.: Subpixel Measurement of 3D Surfaces by Laser Scanning. Sensors and Actuators 76, 1-8 (1999)

12. Sokovic, M., Kopac, J.: RE (Reverse Engineering) as Necessary Phase by Rapid Product Development. Journal of Materials Processing Technology 175(1-3), 398-403 (2006) 